\title{
Implementation of Data Conversion Interface Between Midas and DUT2014 Structural Model
}

\author{
Xu Ji, Zhinan Ren, Zhe Qi, Zheng He* \\ Department of Civil Engineering, Dalian University of Technology, Dalian, China \\ Email address: \\ 2352722345@qq.com (Xu Ji), 1835496990@qq.com (Zhinan Ren), 1163319187@qq.com (Zhe Qi), hezheng@dlut.edu.cn (Zheng He) \\ ${ }^{*}$ Corresponding author
}

To cite this article:

Xu Ji, Zhinan Ren, Zhe Qi, Zheng He. Implementation of Data Conversion Interface Between Midas and DUT2014 Structural Model. Science Discovery. Vol. 7, No. 2, 2019, pp. 126-133. doi: 10.11648/j.sd.20190702.22

Received: April 28, 2019; Accepted: May 20, 2019; Published: May 23, 2019

\begin{abstract}
DUT2014 is a computational program developed by our team for the elastic-plastic time history analysis of super high-rise building structures. The integrated platform code is open source, which has all the materials and elements for the structural analysis of super high-rise buildings. Moreover, the integrated platform has a new element state transition algorithm (STP), which greatly improves the computational efficiency of elastic-plastic time history analysis. Midas is a finite element analysis software, which has good elastic analysis function and non-linear analysis function, and it has good pre-processing function. Preprocessing of DUT2014 is a command stream format in text format, and its modeling function is relatively cumbersome. In order to realize the customization of DUT2014 modeling function, this paper decided to develop an interface program to transform Midas model data file to DUT2014 model data file. This paper establishes an interface program for converting Midas structured data model file into DUT2014 structured data model file by using Microsoft Visual C++ programming program. In order to verify the correctness of structural data model transformation, this paper will use interface program to transform three different scale structures, and then verify the accuracy of the interface by comparing the quality information, modal calculation results, elastic time history response and elastic-plastic time history response of the model before and after transformation.
\end{abstract}

Keywords: Midas, DUT2014, Data Interface, Fiber Beam-Column Element

\section{Midas与DUT2014结构模型数据转化接口的实现}

姬序, 任智楠, 齐哲, 何政"

大连理工大学土木工程学院, 大连, 中国

邮箱

2352722345@qq.com（姬序），1835496990@qq.com（任智楠）, 1163319187@qq.com（齐哲）, hezheng@dlut.edu.cn（何政）

摘要: DUT2014是本课题组为了实现超高层建筑结构弹塑性时程分析开发的一款计算力学程序, 该集成平台代码开源, 具有超高层建筑结构分析的全部材料和单元，而且集成了新的单元状态转换算法（STP），大大的提升了弹塑性时程 分析的计算效率。Midas是一款有限元分析软件, 具有不错的弹性分析功能和非线性分析功能, 而且具有不错的前处理 功能。DUT2014的前处理是文本格式的命令流形式, 建模功能相对繁琐, 为了实现DUT2014建模功能用户化, 本文决 定开发Midas模型数据文件向DUT2014模型数据文件转化的接口程序。本文通过Microsoft Visual C++编写程序建立了 Midas结构数据模型文件转化为DUT2014结构数据模型文件的接口程序。为了对结构数据模型转化的正确性进行验证, 本文将应用接口程序对三个不同规模的结构分别进行模型转化, 然后通过对比转化前后模型的质量信息、模态计算结 果、弹性时程响应和弹塑性时程响应验证接口的准确性。 
关键词: Midas, DUT2014, 数据接口, 纤维梁柱单元

\section{1. 引言}

关于程序的接口工作主要的内容是模型数据文件的 转化, 研究者们对此作了不少的工作。YJK软件实现了 众多软件之间的模型数据文件的接口, 其中包括YJK和 ETABS的接口, YJK和SAP2000的接口, YJK和Midas的 接口, YJK和ABAQUS的接口。为了实现用户对软件的 使用需求, 新版的PKPM开发了自己的接口程序, 其中包 括PKPM与ETABS的接口, PKPM与Midas的接口。陈学 伟做了ETABS与Opensees的模型数据文件的接口转化。 李加成等 [1]研究了ETABS 到ABAQUS 的模型接口数据 文件的转换。文中介绍了模型转化的基本框架, 并通过 算例验证了模型转化接口程序的正确性。董立坤等[2]研 究了PKPM与ETABS模型数据转化接口的实现。文中介 绍了ETABS模型文件 $\mathrm{e} 2 \mathrm{k}$ 文件的数据格式, 介绍了模型转 化的基本流程, 并通过算例验证了接口程序转化的正确 性。孟仲永等[3]研究了SAP2000到ABAQUS的模型数据 转化接口程序, 文中详细介绍了模型转化的关键技术问 题, 并通过算例验证了模型转化接口程序的正确性。杨 先桥等[4]研究了SAP2000到NASTRAN的模型数据文件 接口程序, 文中介绍了SAP2000与NASTRAN数据文件的 关键字, 并介绍了模型数据文件转化中遇到的难点性问 题, 最后通过数值算例验证了模型转化接口程序的正确 性。王文渊[5]研究了SATWE与ETABS模型数据转化的接 口, 文中详细介绍了SATWE与ETABS文本模型的数据结 构的特点, 并且详细描述了带有开口的墙单元在两种软 件中的转化要点, 并通过框架剪力墙结构与框架核心筒 结构验证了模型转化接口程序的正确性。王杰等[6]研究 了YJK到ABAQUS模型数据文件的转化接口, 文中详细 介绍了模型转化接口程序执行过程中的关键技术, 并通 过简单算例和复杂算例验证了模型转化接口程序的正确 性。

DUT2014[7-8]集成平台是本课题组针对超高层结构 分析开发的一款计算力学程序, 该程序具有不错的非线性 分析功能, 而且计算效率相比其他商业软件要高。 Midas[9-11]是一款有限元分析商业软件, 具有不错的弹性 和非线性分析功能，而且具有适合用户建模的前处理界面。 由于DUT2014前处理建模是通过文本格式命令流的形式 进行的, 其建模工程相对比较复杂, 而且不利于用户建模, 为了实现DUT2014的前处理建模用户化, 本文将用 Microsoft Visual C++编写Midas模型数据文件到DUT2014 集成平台建模文件的接口程序, 并通过计算对比模型转化 前后结构的总质量、自振周期、弹性时程响应、弹塑性时 程响应验证模型转化接口的正确性。
本文的主要研究思路是在深入学习 $\mathrm{C}++$ 字符串处理 和有限元分析基本参数设置的基础上, 编写文本转化的接 口程序, 其主要研究难点是攻克接口转化的技术性问题, 这些技术性问题包括模型信息的对应策略、正确建立ep文 件材料、截面与单元的映射关系和正确确定杆件截面方向, 编写完接口程序后通过结构模型对模型转化的正确性进 行验证。

\section{2. 接口转化过程介绍}

\section{1. 节点转化}

节点转化的主要任务是将Midas Gen MGT文件中节 点格式转化成DUT2014中的节点格式, 节点转化的主要任 务是读取Midas Gen MGT文件中的节点号和节点坐标, 然 后按照ep文件的格式输出到该文件中。

\section{2. 单元转化}

单元转化主要分为两部分, 框架单元转化和壳单元转 化。Midas中的框架单元包括两种, 一种是桁架单元, 一 种是一般梁/变截面梁单元, DUT2014的框架单元包括弹 性梁单元ElasticBeam3d、基于位移的纤维梁柱单元 DispBeamColumn、基于力的纤维梁柱单元 ForceBeamColumn, 本文做接口程序主要是对一般梁/变截 面梁单元向基于位移的纤维梁柱单元的转化; Midas中的 壳单元包括板单元、平面应力单元、平面应变单元、墙单 元, DUT2014中的壳单元包括弹性壳LayeredShell和弹塑 性壳LayeredShellGNL, 本文做的接口程序是将板单元与 墙单元向弹塑性壳LayeredShellGNL进行接口转化。选取 这样的单元转化策略是为了对转化后的数据模型更好的 进行非线性分析。

\section{3. 材料转化}

Midas的材料总共有三种, 钢材、混凝土材料和组合 材料, 而DUT2014的材料也有三种, 分别为ConcreteKP、 SteelGMP和Mcft材料, Midas的钢材材料对应于DUT2014 的SteelGMP材料, Midas的混凝土材料对应于DUT2014的 ConcreteKP材料和 Mcft材料, Midas的组合材料对应于 DUT2014的SteelGMP材料和ConcreteKP材料。下图1将给 出材料转化的详细流程图。 


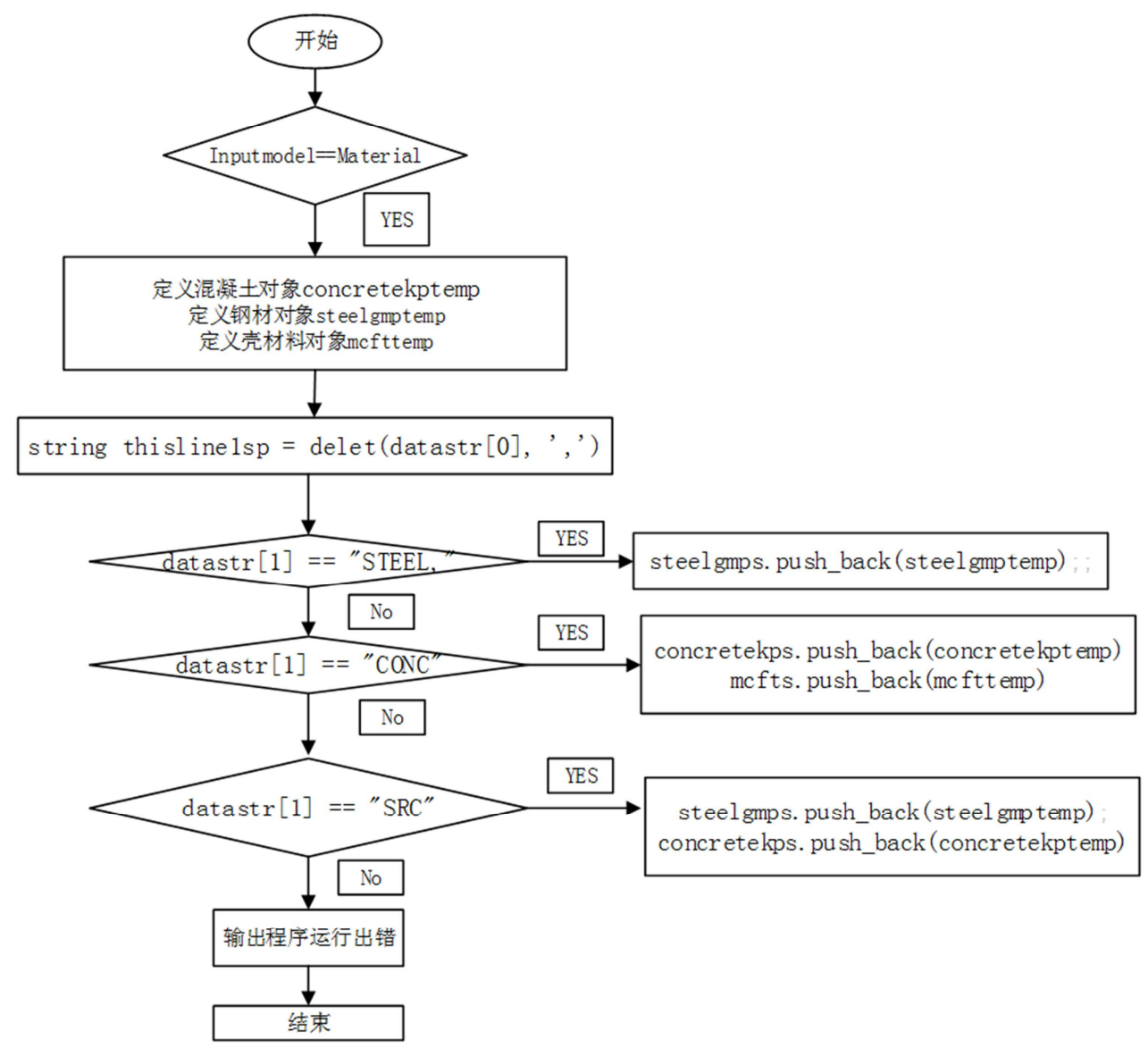

图1 材料转化流程图。

\section{4. 纤维截面转化}

Midas的截面分为两种一种是型钢截面、一种是组合 截面, 而DUT2014的纤维截面则通过组合式进行截面定义, 首先用FiberSection命令创建空白截面, 然后用AddRect命 令加入矩形截面, 用AddLayer命令加入一排纤维, 用 AddCircle命令加入圆环截面, 同时DUT2014创建了箱型截 面 addBoxSection、角钢截面 addLSection、工字形截面 addWSection等快捷截面形式。总之纤维截面转化就是建 立Midas截面库与DUT2014纤维截面之间的对应关系。

\section{5. 壳截面转化}

壳截面转化主要是对Midas中的壳单元信息转化成 DUT2014集成平台所需的壳单元信息, Midas中的壳截面 参数主要是材料和截面厚度, 本文将通过建立壳截面类将 其转化成DUT2014集成平台所需的壳单元截面形式, 由于 DUT2014集成平台的壳截面具有钢筋信息, 该信息无法从 Midas中读取, 故该信息转化之后将由用户自己改动。

\section{6. 质量转化}

Midas的质量定义选项包括节点质量、刚性楼板质量, 以及将荷载转化成质量, 而按照质量控制参数划分分为集
中质量和一致质量, 而对于DUT2014的质量是采用集中质 量的方法来考虑质量的。在质量转化的过程中, 质量主要 分为两种, 一种是恒荷载转化成的质量, 一种是活荷载转 化成的质量, 其中恒荷载转化成的质量来自于结构自重和 附加恒荷, 活荷载的质量来自于结构附加的活荷。具体的 质量计算采取均分的方法, 梁柱的节点质量按照单元的长 度进行对半分, 各取一半, 壳单元的节点质量按照四个节 点各取 0.25 进行计算。

\section{3. 接口转化的关键技术性问题}

\subsection{Midas与DUT2014模型信息的对应策略}

该关键技术主要是为了实现Midas与DUT2014模型信 息的对应, 故而研究了Midas导出的mgt文件的数据模型 关键字与DUT2014的 ep 文件的数据模型关键字。通过 Midas的mgt文件的数据模型关键字可将文件读取的过程 分成节点、材料、单元等若干块, 然后分块读取信息, 读 入信息全部存入对应的vector $(\mathrm{C}++)$ 容器之后, 然后按照 DUT2014所需的ep文件中的关键字进行正确写出, 实现模 型转化的有序正确进行。 
表1 Midas与DUT2014模型信息关键字映射。

\begin{tabular}{lll}
\hline Midas关键字 & DUT2014关键字 & 备注 \\
\hline *NODE; Nodes & Node & 节点定义 \\
*ELEMENT; Elements & DispBeamColumn & 单元定义 \\
& LayeredShellGNL & 材料定义 \\
*MATERIAL; Material & SteelGMP & 框架截面定义 \\
*SECTION; Section & ConcreteKP & 墙板截面定义 \\
*THICKNESS; Thickness & addBoxSection & 约束定义 \\
*CONSTRAINT; Supports & ShellSection & \\
\hline
\end{tabular}

\section{2. ep文件材料、截面与单元之间的映射关系的形成}

由于DUT2014的ep文件里的材料信息、截面信息和单 元信息存在内在的映射关系, 故而进行接口程序编写的时 候, 其关键技术是正确建立三者之间的映射关系, 才能保 证模型数据接口的正确。为了实现材料信息与截面信息的 正确映射关系, 在建立框架类时需要读入 mgt文件中具备 的原始材料编号与原始截面编号, 在建立材料类时需要读 入mgt文件中的原始材料编号, 然后在将材料信息赋予截 面信息时，通过框架类找到截面与材料的对应关系。为了 实现截面信息与单元信息之间的正确映射关系, 接口程序 建立了寻找线截面编号的 searchLineNum函数与寻找面截 面编号的searchAreaNum函数, 通过这两个函数找到框架 单元与壳单元所需的截面编号, 将其赋予框架截面信息与 壳截面信息。实现了这两种映射关系的正确建立, 接口转 化程序的主要技术性难点便攻克了。

\section{3. 杆件截面方向的确定}

杆件截面方向的确定是接口程序需要解决的又一个 关键性技术问题, 下面分别给出了Midas与DUT2014确定 杆件截面方向的方法。Midas是通过截面定位角 $\beta$ 来确定杆 件的截面方向的, 而DUT2014是通过截面定位向量来确定 杆件的截面方向的, 接口程序需要解决的关键技术是找到 截面定位向量角 $\beta$ 与截面定位向量 $\{g t x, g t y, g t z\}$ 之间的关系。 具体的通过研究找到了两者之间的对应关系, 该关系将在 以下部分中公式(1)和(2)给出。

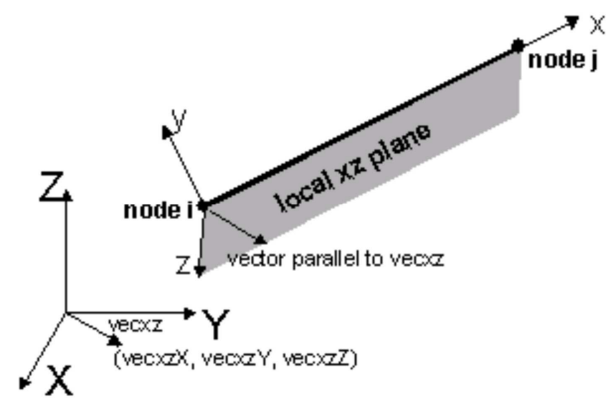

图2 DUT2014截面定位向量确定

坚向构件时:

$$
\left\{\begin{array}{l}
g t x=\cos (\beta) \\
g t y=\sin (\beta) \\
g t z=0
\end{array}\right.
$$

水平构件或斜撑时:

$$
\left\{\begin{array}{l}
g t x=\sin (\beta) \\
g t y=0 \\
g t z=\cos (\beta)
\end{array}\right.
$$

通过该关系即可建立截面方向角 $\beta$ 与截面定位向量之 间的关系。

\section{4. 模型转化接口的正确性验证}

为了对接口程序的模型转化正确性进行全面的验证, 本文将使用各种类型各种规模的结构对模型转化接口进 行测试和验证, 下面使用小结构框架、中结构框架和大结 构框架核心筒三个典型工程实例模型对模型转化接口程 序进行验证, 通过小结构验证混凝土材料结构转化的正确 性, 通过中结构验证钢结构转化的正确性, 通过大结构对 各种复杂截面情况下的导模型正确性进行验证, 在验证响 应方面本文选取了结构的前 12 阶自振周期、结构的总质量、 结构的弹性时程响应与结构的弹塑性时程响应对模型转 化的正确性进行了验证进行了验证。

图3给出了小结构、中结构与大结构三个模型的详细 信息。大结构为框架核心筒结构, 梁单元共采取了12种工 字形截面, 柱单元总共采取了 8 种钢管型硂截面和矩形硂 十字 $\mathrm{H}$ 型钢截面, 剪力墙采取了以米为单位的 $0.13 、 0.2$ 、 $0.6 、 0.7 、 0.8 、 1$ 等六种厚度。为了进行小震和大震下的 顶点位移时程响应验证, 分别对三种形式的结构进行了弹 性时程分析与弹塑性时程分析, 分析时选取名为 RH4TG055[12]的地震波进行分析, 弹性时程分析时通过 调幅将地震波幅值调制 $70 \mathrm{~cm} / \mathrm{s}^{2}$, 弹塑性时程分析时通过调 幅将地震波幅值调制 $400 \mathrm{~cm} / \mathrm{s}^{2}$, 地震波的波形图在下图4 中给出。结构弹塑性分析的详细信息将在下文给出。

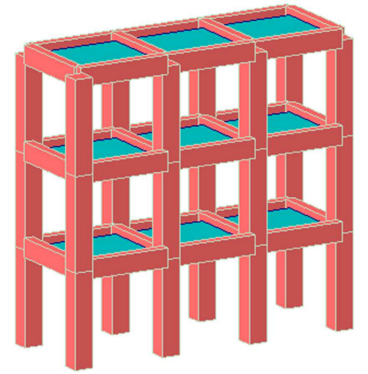

楼层数: 3

层高: $3 \mathrm{~m}$

节点数: 31

纤维梁柱单元数: 54

分层壳单元数: 9

截面类型: 矩形

混凝土材料: C30

(a) 小结构模型图 


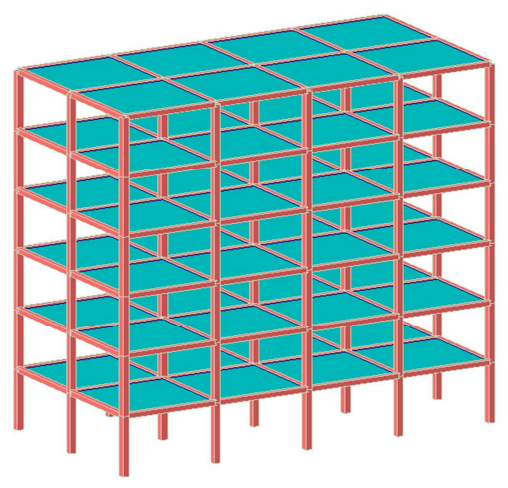

(b) 中结构模型图

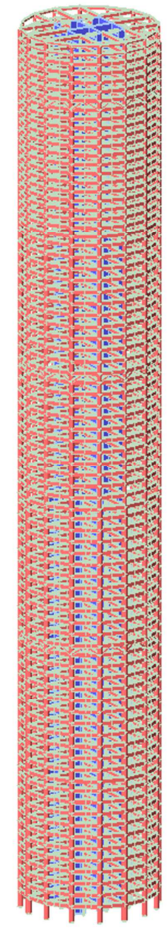

楼层数: 81

层高： $3.3 \mathrm{~m} 、 4.2 \mathrm{~m}$

结构总高: $318.6 \mathrm{~m}$

节点数: 7172

纤维梁柱单元数: 9279

分层壳单元数: 1728

大结构里的柱截面形式:

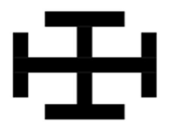

大结构里的梁截面形式:

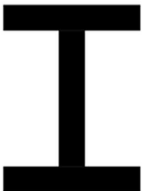

(c) 大结构模型图

图3 结构模型图。

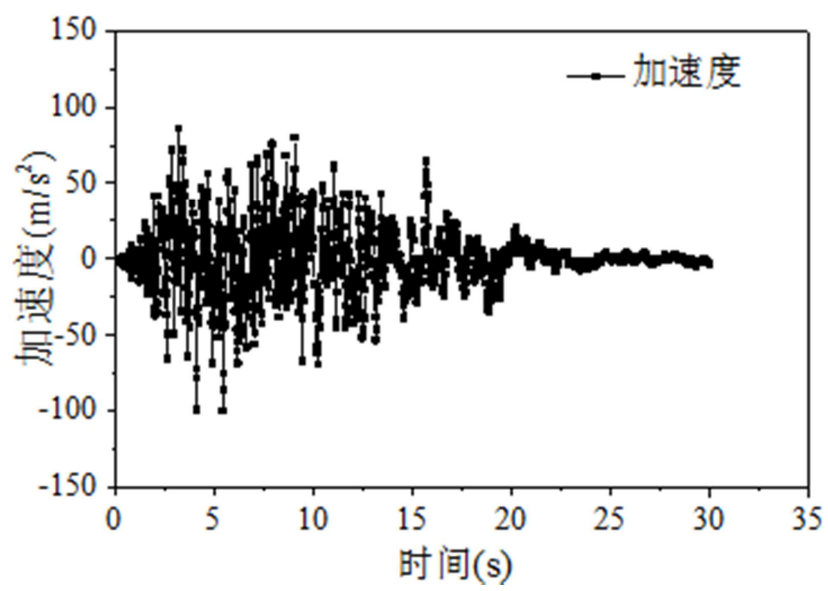

图4 地震波图。

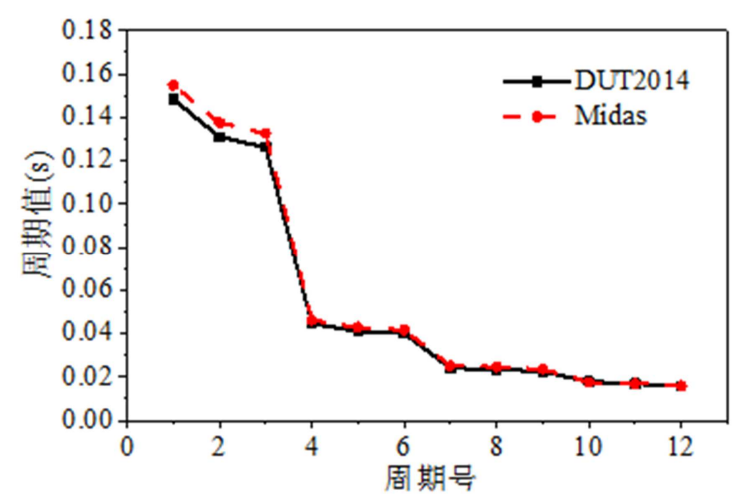

图5 小结构周期对比。

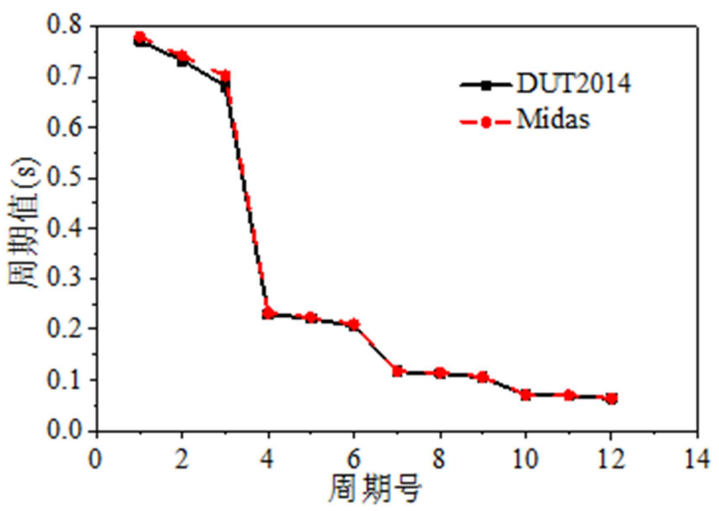

图6 中结构周期对比。

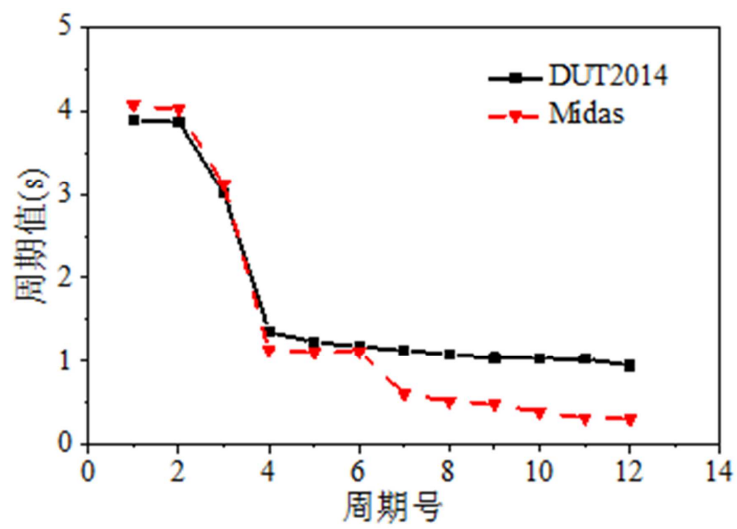

图7 大结构周期对比。

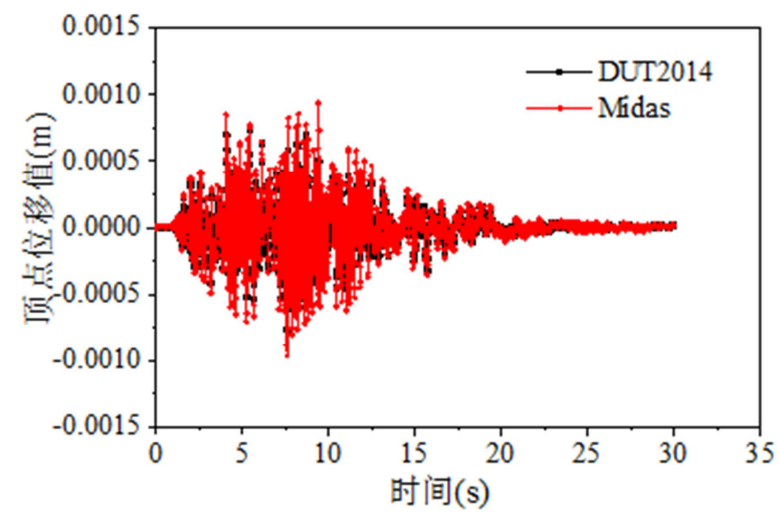

图8 小结构弹性响应对比。 


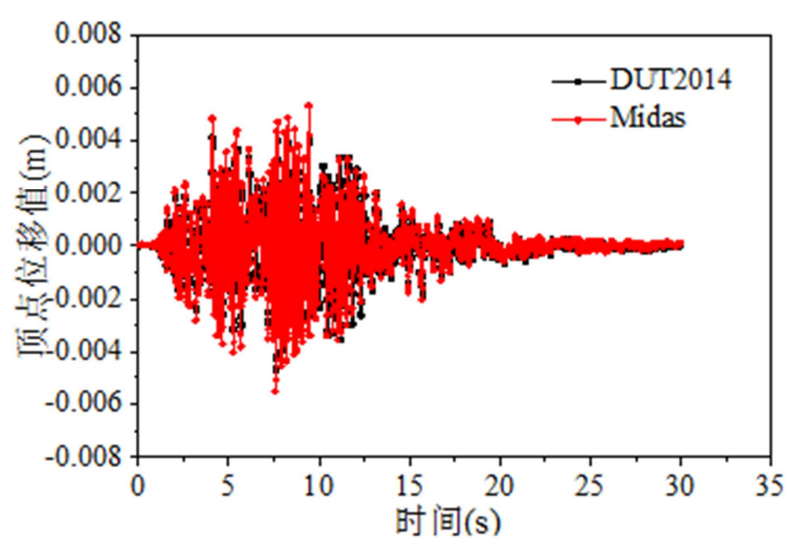

图9 小结构弹塑性响应对比。

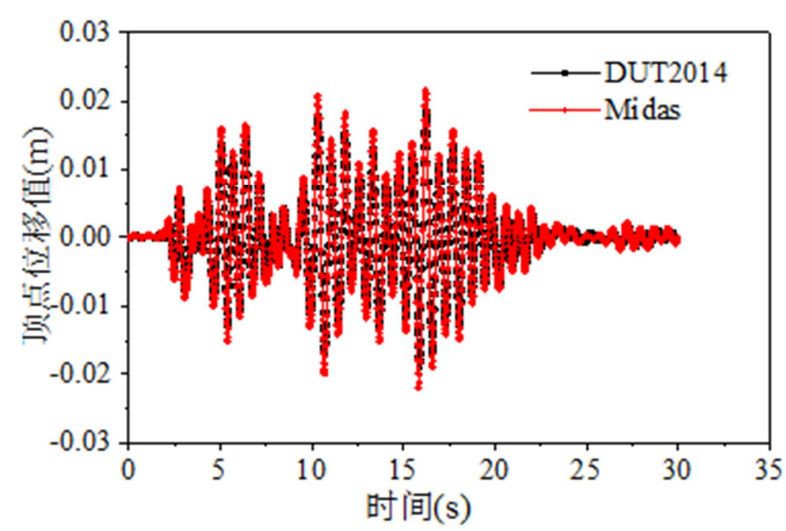

图10 中结构弹性响应对比。

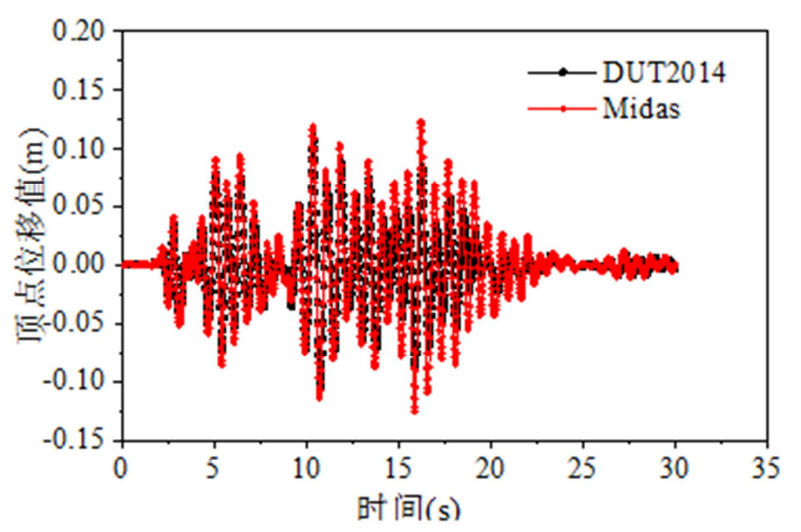

图11 中结构弹塑性响应对比。

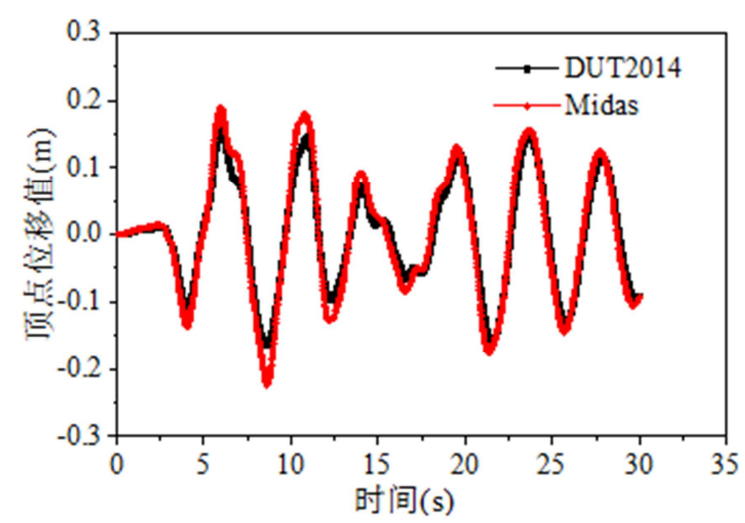

图12 大结构弹性响应对比。

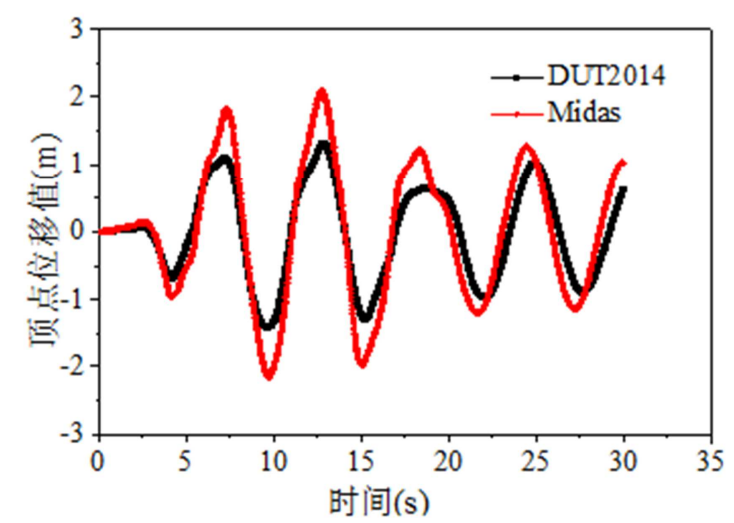

图13 大结构弹塑性响应对比。

表2 结构总质量对比 $\left(\times 10^{3} \mathrm{~kg}\right)$ 。

\begin{tabular}{llllll}
\hline 小结构 & & 中结构 & & 大结构 \\
\hline DUT2014 & Midas & DUT2014 & Midas & DUT2014 & Midas \\
\hline 132.19 & 132.16 & 803.23 & 803.62 & 123780 & 123601 \\
\hline
\end{tabular}

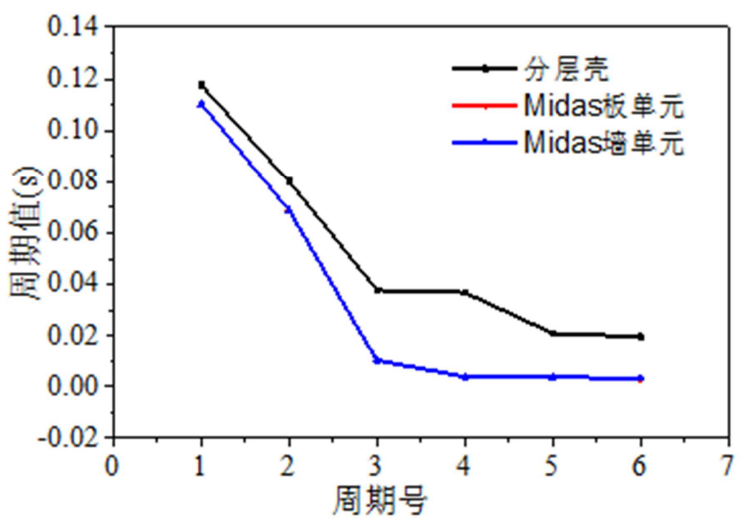

图14 壳单元属性对比图。

表3 大结构所用截面单柱测试。

\begin{tabular}{llll}
\hline 截面类型 & 周期号 & Midas周期(sec) & DUT2014周期(sec) \\
\hline \multirow{2}{*}{ RH2T截面 } & 1 & 0.0557 & 0.055598 \\
& 2 & 0.0526 & 0.052066 \\
& 3 & 0.0032 & 0.015683 \\
矩形截面 & 1 & 0.0748 & 0.076714 \\
& 2 & 0.0378 & 0.038293 \\
& 1 & 0.0037 & 0.013357 \\
工字形截面 & 2 & 0.0675 & 0.069115 \\
& 3 & 0.0151 & 0.014034 \\
钢管混凝土截 & 1 & 0.0026 & 0.008491 \\
面 & 2 & 0.0311 & 0.03129 \\
\hline
\end{tabular}

在弹塑性时程分析中, DUT2014程序的混凝土本构为 Kent-Park模型[13], 钢材本构为SteelGMP模型, 对于Midas 的弹塑性时程分析, 小结构的混凝土本构采取了混凝土设 计规范 (GB50010-02) 里的本构, 中结构钢材本构选取了 Menegotto-Pinto模型[14], 大结构采用非弹性铰模型考虑 构件的非线性, 所选取的滞回模型为随动强化型滞回模型。

对三种结构的前 12 阶自振周期、结构总质量、结构弹 性顶点位移时程、结构弹塑性顶点位移时程进行了对比, 通过对比发现三种结构的各种响应基本上是吻合的。当讨 论小结构响应时, 通过图5 可以看出小结构前 12 阶周期基 
本是吻合的，通过表 2 可以看出小结构的总质量也是吻合 的, 从而可以大体的得出小结构的刚度矩阵和质量矩阵是 一致的，再通过图8、图9可以看出小结构的时程响应结果 是吻合的, 从而可以进一步判断小结构的刚度、质量矩阵 是吻合的，小结构在DUT2014与Midas中的非线性材料属 性是吻合的, 从而初步验证了混凝土结构模型接口转化的 正确性。

当讨论到中结构时, 通过图6可以看出, 中结构的前 12 阶周期是吻合的, 通过表 2 可以看出, 中结构的总质量 是吻合的，同样的再通过图10、图11我们可以看出，中结 构的弹性及弹塑性响应也是吻合的, 从而可以判断出中结 构的刚度、质量矩阵是吻合的，材料的非线性属性也是吻 合的, 从而初步验证了钢结构模型转化接口的正确性。当 讨论到大结构响应时, 从图7可以看出其前六阶自振周期 基本上是吻合的，后面的周期出现了偏差，通过表 2 可以 看出大结构的总质量是吻合的, 由此可以判断出大结构周 期差异来自于结构刚度矩阵。

为了寻找周期出现偏差的原因, 通过截面的单柱测试 依次测试了大结构使用的截面，通过表3发现截面的周期 响应基本上是吻合的, 大致可以排除周期出现偏差是由杆 梁单元造成的, 而但对剪力墙进行测试时, 通过图14可以 发现Midas里的墙单元和DUT2014里的墙单元存在差异, 所以初步估计周期出现偏差的原因是由剪力墙造成的。造 成这种差异的原因可能是是DUT2014里的分层壳单元 [15-17] 与Midas里的板单元与墙单元的单元属性不同。 DUT2014里的分层壳单元是由平面膜元和平面板元组成, 每个节点考虑六个自由度, 同时考虑几何非线性在合成壳 单元刚度矩阵的时候加入了几何非线性刚度矩阵。而 Midas里的板单元每个节点考虑五个自由度, 没有考虑单 元的面内扭转, 在节点自由度方面与分层壳单元有差别, 而Midas里的墙单元每个节点考虑五个自由度, 没有考虑 垂直于板面的位移自由度, 在节点自由度方面也与分层壳 单元有区别。在单元材料方面DUT2014的壳单元采用 Mcft 二维材料 [18], 而Midas采用普通的混凝土材料。

通过图12、图13对比大结构的弹性顶点位移响应和弹 塑性顶点位移响应, 发现弹性响应基本上是一致的, 由此 可以判断大结构的初始刚度和初始质量是一致的, 而对于 弹塑性时程响应的幅值出现偏差, 其可能的原因是 DUT2014选取纤维梁柱单元考虑非线性, 而Midas采用非 弹性铰模型考虑非线性。

\section{5. 结论}

本文主要介绍了商业软件Midas数据模型向计算力学 程序DUT2014的接口转化, 接口转化主要是利用了 Microsoft Visual C++强大的字符串处理功能, 具体的接口 程序以Midas导出的Midas Gen MGT文件为输入文件, 将 其转化成DUT2014所需的ep文件。模型接口程序充分发挥 了Midas的前处理功能, 避免了DUT2014繁琐的命令流建 模, 为实现利用计算力学程序DUT2014进行超高层结构弹 塑性时程分析打好了坚实基础。

\section{致谢}

感谢何政老师在该篇论文写作过程中的精心指导。

\section{参考文献}

[1] 李加成, 钟双玲. ETABS到ABAQUS转换程序开发 [C]// 第 十二届全国现代结构工程学术研讨会暨第二届全国索结构 技术交流会。

[2] 董立坤, 王徽. PKPM与ETABS结构模型数据转换接口的 实现[J]. 土木建筑工程信息技术, 2012(3)。

[3] 孟仲永, 张慎, 王杰. SAP2000到ABAQUS的模型转换程序 研究与开发 [J]. 建筑结构, 2017(s2):552-555。

[4] 杨先桥, 傅学怡, 黄用军. SAP2000 到 ABAQUS 及 NASTRAN的模型转换程序SAPTRANS介绍[J]. 建筑结构, 2010(11):I0008-I0011。

[5] 王文波. SATWE与ETABS数据接口软件编制[J]. 工程建设 与设计, 2008(z1):42-47。

[6] 王杰, 张慎. 复杂高层建筑从YJK到ABAQUS的模型转换 程序研究与开发 [J]. 建筑结构, 2017(S2):546-551。

[7] 付少俊. 超高层建筑结构动力弹塑性时程分析加速算法与 集成[D]. 大连: 大连理工大学, 2016。

[8] Shared Memory Parallel Computing Procedures for Nonlinear Dynamic Analysis of Super High-rise Buildings $[\mathrm{C}]$. Fu SJ, He Z, Li ZQ, Tu X, Tao Q. Second International Conference on Performance-based and Life-cycle Structural Engineering (PLSE 2015), Brisbane, Australia, December 2015.

[9] 侯高峰, 王建国, 张茂. 基于MIDAS/GEN高层建筑结构静 力弹塑性分析 [J]. 合肥工业大学学报 (自然科学版), 2008, 31(10)。

[10] 侯高峰. 基于MIDAS/GEN高层建筑结构三维有限元仿真 分析[D]. 合肥工业大学, 2007。

[11] 陈迎春, 李小飞, 何四祥. MIDAS/Gen在建筑结构施工分 析中的应用研究 [J]. 建筑结构, 2010(s1):504-505。

[12] PEER Ground Motion Database. https: //ngawest2.berkeley.edu/users/sign up.2019.

[13] Kent DC, Park R. Flexural members with confined concrete $[\mathrm{J}]$. Journal of the Structural Division, 1971, 97 (7): 1969-1990.

[14] Menegotto M, and Pinto PE. Method of analysis for cyclically loaded reinforced concrete plane frames including changes in geometry and nonelastic behavior of elements under combined normal force and bending [C]. Proc., IABSE Symposium on Resistance and Ultimate Deformability of Structures Acted on by Well-Defined Repeated Loads, IABSE Reports, Lisbon, Portugal, 1973.

[15] 孙丞江. 应用改进非线性分层壳模型的框架一核心筒结构 强震响应分析[D]. 大连: 大连理工大学, 2017 。 
[16] 何政，孙丞江. 应用改进非线性分层壳模型的超高层框 架——核心筒结构强震响应分析 [J]. 工程力学, 2018, 35(6)。

[17] 须寅, 龙驭球. 采用广义协调条件构造具有旋转自由度的 四边形膜元 $[J]$. 工程力学, 1993, 10(3): 27-36。
[18] Vecchio F J, Collins M P. The Modified Compression Field Theory for Reinforced Concrete Elements Subjected to Shear[J]. AC1 Journal. 1986, 83(2): 219-231。 